\title{
The lipid-chaperon hypothesis: A common molecular mechanism of membrane disruption by intrinsically disordered proteins
}

\author{
Michele F. Sciacca ${ }^{1,9}$, Fabio Lolicato ${ }^{2,3,9}$, Carmelo Tempra ${ }^{4,5,9}$, Federica Scollo ${ }^{5,6,9}$, Bikash \\ R. Sahoo ${ }^{7}$, Matthew D. Watson ${ }^{8}$, Sara García-Vinuales, ${ }^{1}$ Danilo Milardi $^{1}$, Antonio \\ Raudino $^{5}$, Jennifer C. Lee ${ }^{8}$, Ayyalusamy Ramamoorthy ${ }^{7}$ and Carmelo La Rosa ${ }^{5, *}$ \\ ${ }^{1}$ Istituto di Cristallografia, CNR, Catania, Italy \\ ${ }^{2}$ Heidelberg University Biochemistry Center, Heidelberg, Germany \\ ${ }^{3}$ Department of Physics, University of Helsinki, P.O. Box 64, FI-00014 Helsinki, Finland \\ ${ }^{4}$ Institute of Organic Chemistry and Biochemistry, Prague, Czech Republic \\ ${ }^{5}$ Department of Chemical Sciences, University of Catania, Catania, Italy \\ ${ }^{6}$ J. Heyrovský Institute of Physical Chemistry, Academy of Sciences of the Czech Republic, \\ Prague, Czech Republic \\ ${ }^{7}$ Biophysics and Department of Chemistry, University of Michigan, Ann Arbor, Michigan \\ ${ }^{8}$ National Institutes of Health, Bethesda, Maryland
}

${ }^{9}$ These authors contributed equally: Michele F. Sciacca, Fabio Lolicato, Carmelo Tempra, Federica Scollo

\begin{abstract}
Increasing number of human diseases have been shown to be linked to aggregation and amyloid formation by intrinsically disordered proteins (IDPs). Amylin, amyloid- $\beta$, and $\alpha$-synuclein are, indeed, involved in type-II diabetes, Alzheimer's, and Parkinson's, respectively. Despite the correlation of the toxicity of these proteins at early aggregation stages with membrane damage, the molecular events underlying the process is quite complex to understand. In this study, we demonstrate the crucial role of free lipids in the formation of lipid-protein complex, which enables an easy membrane insertion for amylin, amyloid- $\beta$, and $\alpha$-synuclein. Experimental results from a variety of biophysical methods and molecular dynamics results reveal this common molecular pathway in membrane poration is shared by amyloidogenic (amylin, amyloid- $\beta$, and $\alpha$-synuclein) and nonamyloidogenic (rat IAPP, $\beta$-synuclein) proteins. Based on these results, we propose a "lipidchaperone" hypothesis as a unifying framework for protein-membrane poration.
\end{abstract}




\section{Introduction}

Amyloidoses encompass a family of diseases characterized by the misfolding and aggregation of disparate proteins into a common fibrillar, cross- $\beta$-sheet form termed amyloid. The most extensively studied amyloid-forming proteins include: i) the amyloid- $\beta$ (A $\beta$ ) peptide, which is known to form plaques in Alzheimer's disease, ii) $\alpha$-synuclein ( $\alpha$-syn), responsible for Lewy body formation in Parkinson's disease and iii) islet amyloid polypeptide protein (IAPP or amylin), which is linked to type 2 diabetes. All of these proteins are known as Intrinsically Disordered Proteins (IDPs) because they exist as highly dynamic conformational ensembles rather than well-defined folded structures. The correlation between amyloid formation and the development of disease symptoms has given rise to the amyloid hypothesis, which posits that amyloid plaques are responsible for cell death leading to disease progression ${ }^{1}$. The hypothesis is limited, however, by the finding that many patients lack significant plaque build-up; equally problematic is the observation that amyloid deposition occurs even in some healthy individuals ${ }^{2}$. More recently, the toxic oligomer hypothesis, supported by in vivo and in vitro data, suggests oligomers or prefibrillar aggregates rather than the mature and stable fibrils are the species mainly responsible for the cell death leading to disease pathology ${ }^{3}$.

Although there is a broad consensus that membrane damage is an important driver of cellular toxicity in amyloid diseases, the mechanism of the damage remains unsettled. A number of reports concluded that membrane damage is caused by the fibrillar structures themselves ${ }^{4-6}$, whereas the damage mechanism in the toxic oligomer hypothesis has been described as occurring in sequential steps $^{7,8}:$ i) self-assembly of proteins in the lipid phase to form isolated trans-membrane ion-channellike pores (with a diameter of approximately $1.8 \mathrm{~nm}^{8-11}$ ), and ii) a combination of the process of conversion of the assemblies into large aggregates ${ }^{11}$ and membrane-assisted fibrillation process has been shown to tear away lipids from the bilayer ${ }^{7,8}$. Both of these damage mechanisms necessarily assume a first step in which the amyloidogenic protein transfers from the aqueous phase to the hydrocarbon core of the bilayer; this determinant step is still poorly understood. From an experimental point of view, the detection of both small and large pores is mainly based on fluorescent dye leakage assays. Small pores produced by $\mathrm{A} \beta$ are detected using the $\mathrm{Ca}^{2+} / \mathrm{Fura}-2$ pair $^{7}$ while large pores are detected through the release of carboxyfluorescein from the lipid wall ${ }^{8,12}$. The size of these pores is variable and depends on the different amyloidogenic proteins; this is illustrated by the observation that both small and large pores formed by hIAPP and $\alpha$-syn are detectable by the carboxyfluorescein fluorescent probe $^{8}$ and calcein ${ }^{13}$ respectively.

Many biophysical studies have examined IDP-membrane interactions using large unilamellar vesicles (LUVs) as the simplest lipid assembly mimicking the cell membrane. Most literature reports employing LUV model systems focus on lipid-protein interactions occurring in the lipid-rich phase 
(bilayer), while the interactions occurring between proteins and free lipids in the aqueous phase are usually neglected because of the extremely low lipid concentration in water ${ }^{14}$. However, a chemical equilibrium between dispersed lipid monomers and their supramolecular assemblies (LUVs) exists and is characterized by the critical micellar concentration (CMC). For lipid molecules with short acyl chains or charged head-groups, the concentration of free lipids in equilibrium with LUVs may reach values up to the $\mu \mathrm{M}$ range, while the $\mathrm{CMC}$ of long acyl chain containing lipids drops to low $\mathrm{nM}^{15,16}$. Since in most experiments assaying amyloid/membrane interactions, proteins are present at $\mu \mathrm{M}$ concentrations, it is plausible that a free lipid-protein binding equilibrium may also exist in the aqueous phase and that it may influence the insertion rate of proteins into the lipid bilayer. Recently, some of us have developed a phenomenological model simulating the transfer kinetics of a lipidprotein complex from the aqueous phase to the lipid bilayer core ${ }^{17}$. According to this model, watersoluble lipid-protein complexes penetrate the membrane faster than the bare protein provided the hydrophobicity of the lipid-protein complex is higher than that of the bare protein. Both biophysical experiments and molecular simulations carried out on human IAPP (hIAPP) consistently supported this hypothesis, demonstrating the key role played by free lipids in driving membrane poration mechanisms and membrane-bound fibril formation ${ }^{16}$.

Here, we tested the generality of lipid-assisted penetration of amyloidogenic IDPs into membranes by investigating two other amyloidogenic proteins: $A \beta$ and $\alpha$-syn. In addition, we studied rat-amylin and $\beta$-syn as non-amyloidogenic protein controls. In particular, we carried out membrane leakage experiments as a function of different lipid chain lengths, with CMC values ranging from $\mathrm{nM}$ to $\mu \mathrm{M}$. The formation of the hypothesized water-soluble lipid/peptide complexes was confirmed by 2D NMR, circular dichroism (CD)-spectroscopy, molecular dynamics (MD) simulations and isothermal titration calorimetry (ITC) measurements using the A $\beta$ peptide as a paradigm of IDPs. The data demonstrate the existence of a stable complex with increased hydrophobicity, essential prerequisites to the lipid-assisted protein transport. The whole of data collected promptly inspired us to propose a general lipid-chaperon hypothesis that is able to explain in a unique framework many unresolved aspects of the biophysics of amyloid-membrane interactions including the role of lipid composition, peptide hydrophobicity/amyloidogenic propensity and the plausible effects of reactive oxygen species (ROS) in activating protein-induced membrane damage. In conclusion, our data show that key players in membrane damage are the lipid-proteins complexes rather than the bare proteins. Moreover, the lipid-chaperone hypothesis appears as a more general molecular model that includes both amyloid and toxic oligomer hypotheses. 


\section{Results}

\section{Free lipids in solution drive the interaction of $A \beta_{1-40}$ with LUVs.}

$\mathrm{A} \beta_{1-40}$ damages model membranes through a two-step mechanism ${ }^{7}$ : in the first step, the oligomeric protein inserts into the bilayer hydrocarbon core forming ion-channel-like pores; in the second step, protein damages the membrane through a detergent-like mechanism, stripping away lipids from the bilayer and forming larger breach than those formed in the first step. To detect these different types of pores, two structurally different fluorescent probes were used: the $\mathrm{Ca}^{2+} / \mathrm{fura}-2$ pair to detect the formation of small pores (ion-channel-like), and carboxy-fluorescein to monitor the formation of large pores. Discrimination of pore dimension arises from the much smaller hydrodynamic radius of $\mathrm{Ca}^{2+}$ compared to that of carboxy-fluorescein.
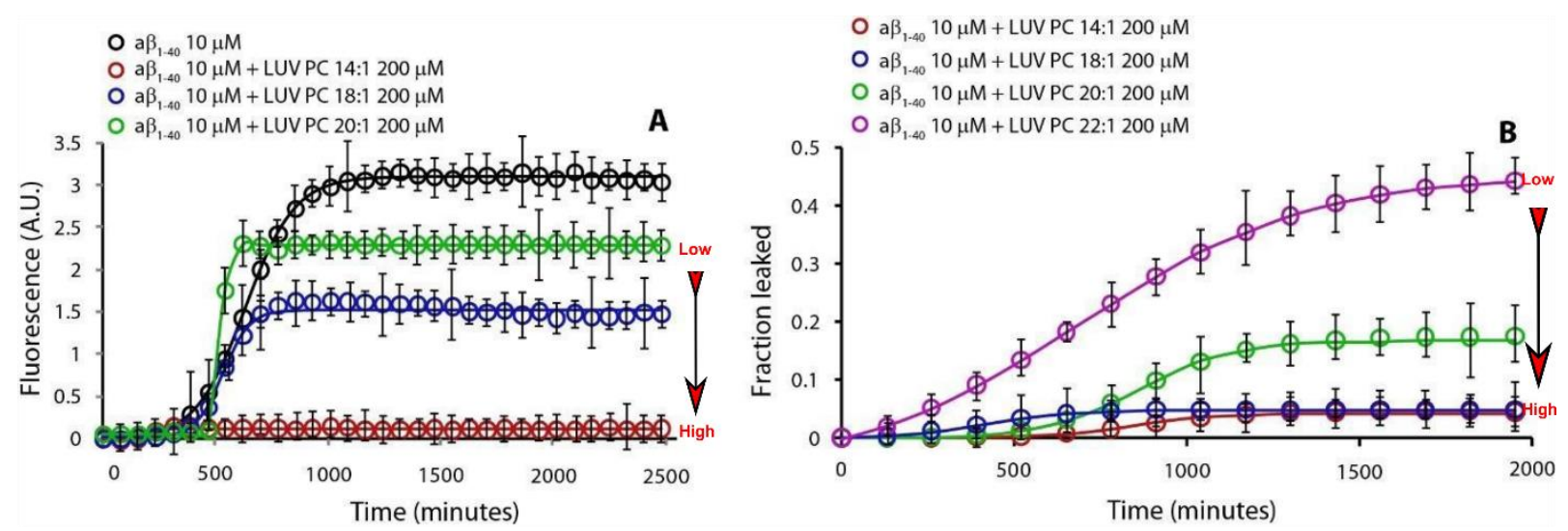

Figure 1. Effect of lipid's hydrocarbon chain length on A $\beta_{1-40}$ fibril formation kinetics (A) and A $\beta_{1-40}$ induced dye leakage (B). (A) ThT traces and (B) dye-leakage for samples containing $10 \mu \mathrm{M} A \beta_{1-40}$ (black, no lipids) in the presence of $200 \mu \mathrm{M}$ LUVs of PC lipids having acyl chains of 14:1 (red), 18:1 (blue) and 20:1 (green). Experiments were performed at $37{ }^{\circ} \mathrm{C}$ in $10 \mathrm{mM}$ phosphate buffer, $100 \mathrm{mM} \mathrm{NaCl}, \mathrm{pH}$ 7.4. All results are the average of three experiments.

Fig. 1A shows $A \beta_{1-40}$ fibril growth kinetics monitored by the thioflavin $T$ (ThT) binding assay in the presence of diacyl-phosphatidylcholine LUVs composed of lipids with different hydrocarbon chain lengths. Specifically, each lipid single chain contains 14,18 or 20 carbon atoms, with CMC values of $1 \times 10^{-7} \mathrm{M}, 2 \times 10^{-8} \mathrm{M}$ and $6.3 \times 10^{-9} \mathrm{M}$, respectively ${ }^{16}$. As the CMC increases from $\mathrm{C} 20$ to $\mathrm{C} 14$, the formation of fibrils is reduced, similar to previous data on hIAPP ${ }^{16}$. On increasing the CMC, the increased content of free lipids in solution interferes with amyloid growth likely because the free lipids interact with hydrophobic monomeric proteins to form stable protein-lipid complexes, as reported for hIAPP (see the lipid-protein complex section below). Indeed, this process is expected to 
be in competition with protein-protein oligomerization, as evidenced by the molecular dynamics simulations and NMR results reported below.

Fig. 1B shows the release of a fluorescent dye (carboxy-fluorescein) from the lumen of LUVs formed by lipids of different acyl chain lengths in the presence of $A \beta_{1-40}$. As the number of carbon atoms increases (i.e. the free lipid concentration decreases), we observe that the release of the dye increases. This appears to be in direct contradiction to the trend observed for hIAPP-induced leakage, where longer-chain, lower-CMC lipid LUVs were more resistant to leakage. However, due to the slow kinetics of the process in Figure 1B, we believe that the effect stems from membrane disruption by a detergent-like mechanism rather than stable ion-channel-like pore formation ${ }^{7,8}$. Since the large size of carboxyfluorescein may prevent the observation of small ion-channel-like pores, we employed the $\mathrm{Ca}^{2+} / \mathrm{fura}-2$ pair as an alternative fluorescent probe. In this assay the fura- 2 dye is trapped in the lumen of the LUVs while the $\mathrm{Ca}^{2+}$ ions are outside. In this configuration $\mathrm{Ca}^{2+}$ and fura- 2 ions do not interact because they are separated by the bilayer. If ion-channel-like pores are formed upon the addition of $\mathrm{A} \beta_{1-40}$ monomers, $\mathrm{Ca}^{2+}$ ions can cross the bilayer and interact with fura- 2 in the lumen of LUVs by forming a detectable fluorescent complex. Indeed, Fig. 2 shows that in the presence of $\mathrm{A} \beta_{1-40} \mathrm{Ca}^{2+}$ enters the lumen of the LUVs, indicating the formation of ion-channels-like. It is evident that lipids with a lower number of carbon atoms favor the formation of ion-channel-like pores in the first stage as detected using $\mathrm{Ca}^{2+} /$ Fura 2 fluorescent pair and large pores in the second stage as detected by using carboxyfluorescein leakage, while lipids with longer acyl chains repress their formation. This behavior has been previously observed for hIAPP and attributed to the presence of free phospholipids in the aqueous phase that are in dynamic equilibrium with those embedded in the membrane ${ }^{16}$. For IAPP, it has been observed that free lipids in solution form a lipid-protein complex that may facilitate the insertion of proteins into the lipid bilayer resulting in the formation of pores. Provided the lipidprotein complexes is more hydrophobic than the bare protein (as discussed later based on results shown in Fig. 10), the complex formation is a needed step for protein penetration into the lipid bilayer $^{17}$. 


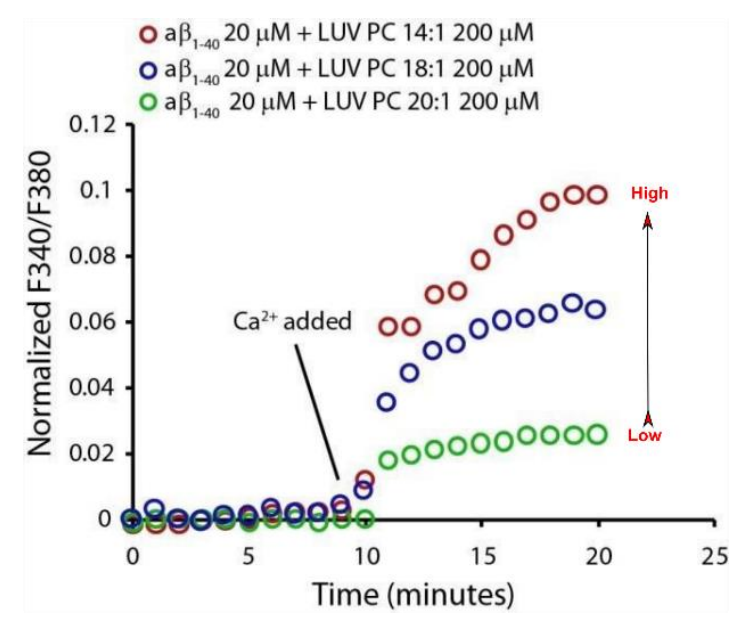

Figure 2. The increase in the hydrocarbon acyl chain length progressively inhibits pore formation. Fura-2 assay indicates that increasing hydrocarbon acyl chain length inhibits pore formation by $20 \mu \mathrm{M} \mathrm{A} \beta 1-40$ on $200 \mu \mathrm{M}$ LUVs of PC 14:1 (red), 18:1 (blue) and 20:1 (green). Experiments were performed in $10 \mathrm{mM}$ HEPES buffer, $100 \mathrm{mM} \mathrm{NaCl}, 200 \mathrm{mM}$ EGTA, pH 7.4.

Results reported in Fig. 2 demonstrate that lipids, with long acyl chains and therefore a small CMC, are more resistant to ion channel-like pore formation by $\mathrm{A} \beta_{1-40}$. This is consistent with the model in which membrane poration is dependent on the formation of lipid-protein complexes in the aqueous phase. In the absence of this complex, the only significant event is the growth of fibrils on the membrane surface and the consequent membrane damage by a detergent-like mechanism as shown in Fig. 1B and Fig.2 respectively.

Free lipids drive the interaction of a-synuclein with LUVs.

We have shown that the relationship between $A \beta_{1-40}$, LUVs and free lipids resembles what previously reported for hIAPP ${ }^{16}$. However, it is not known whether this behavior is common to other amyloidogenic proteins. To examine this, we carried out experiments on $\alpha$-syn, a 140 amino acid protein involved in Parkinson's disease. Fig. 3A shows $\alpha$-syn fibril growth kinetics in the presence of LUVs composed of phospholipids of different acyl chain lengths. As for A $\beta_{1-40}$ and hIAPP, high concentrations of free phospholipids (PC 14:1) in solution abolish the formation of fibrils although the kinetics of synuclein amyloid formation is slower than for $\mathrm{A} \beta_{1-40}$ and hIAPP. 

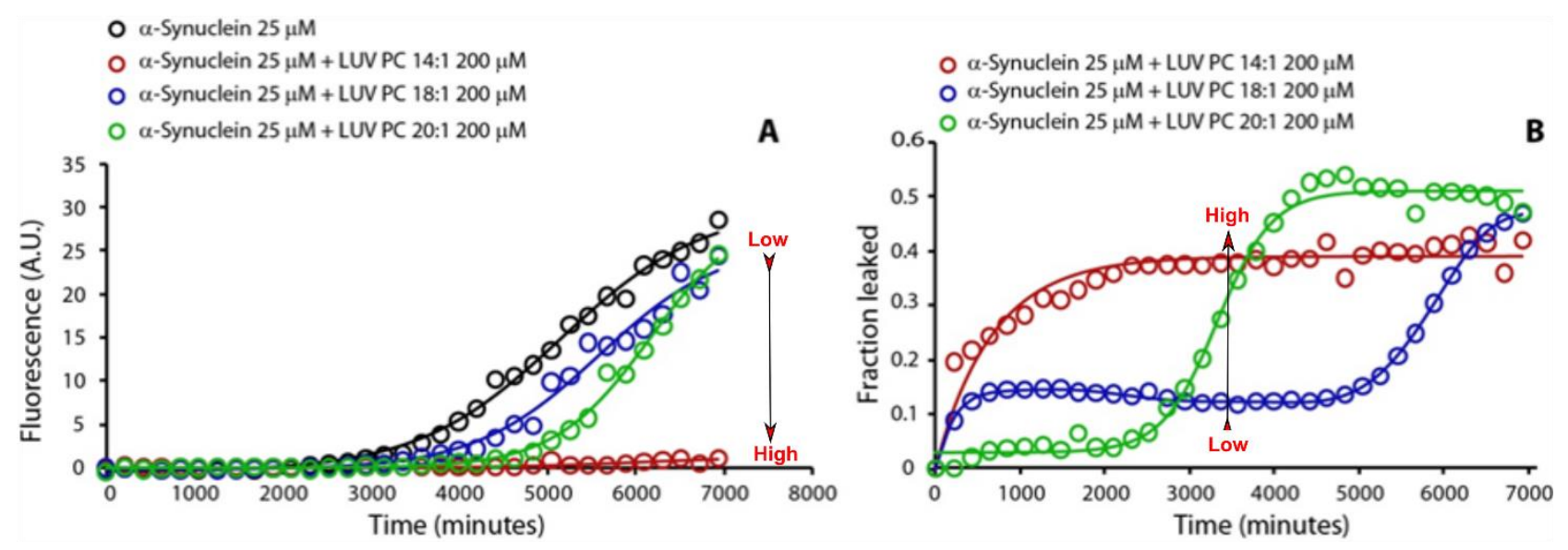

Figure 3. (A) Effect of lipid's hydrocarbon acyl chain length on $\alpha$-syn amyloid formation kinetics (A) and dye-leakage (B). (A) ThT traces and (B) dye-leakage from samples containing $25 \mu \mathrm{M} \alpha$-syn (black curve) in the presence of $200 \mu \mathrm{M}$ LUVs of PC 14:1 (red), 18:1 (blue) and 20:1 (green). Experiments were performed at $37^{\circ} \mathrm{C}$ in $10 \mathrm{mM}$ phosphate buffer, $100 \mathrm{mM} \mathrm{NaCl}, \mathrm{pH}$ 7.4. Solid line represents the best-fit. All results are the average of three experiments.

Fig. 3B shows the kinetics of pore formation monitored by carboxyfluorescein release from LUVs composed of phospholipids with lipid acyl chains of different lengths. Like A $\beta_{1-40}$ and hIAPP, $\alpha$-syn induces pore formation in PC LUVs, but unlike the $A \beta_{1-40}$ it rapidly forms pores large enough to be observed by the carboxyfluorescein release assay. Similar to hIAPP, when $\alpha$-syn interacts with LUVs formed by phospholipids characterized by a low $\mathrm{CMC}$ the rapid formation of pores is not evident; by contrast when $\alpha$-syn interacts with LUVs composed of lipids with a high CMC, pore formation is favored at the expenses of the slower detergent-like mechanism. In the case of intermediate acyl chain length, the two mechanisms are found to be operative. Collectively, the data for IAPP, A $\beta_{1-40}$ and $\alpha$ syn show that the concentration of free phospholipids is a discriminating factor in the interaction of these amyloidogenic IDPs with LUVs, promoting or repressing either pore-formation or detergentlike mechanisms. In particular, the absence of free phospholipids favors the formation of fibrils through a self-assembly process, while high free phospholipid concentrations promote the formation of ion-channel-like pores and detergent-like membrane damage while repressing fibrillogenesis.

\section{The lipid-chaperone hypothesis}

The insertion of a protein in the bilayer core is related to the formation of lipid-protein complexes in solution by simultaneous chemical equilibria:

$$
\begin{array}{ll}
L_{L U V S} \leftrightarrow L & \text { (a) } \\
n P \leftrightarrow P_{n} & \text { (b) }
\end{array}
$$




$$
L+P \leftrightarrow L P \quad(c)
$$

where $L_{L U V S}$ is the number of self-assembled phospholipids in the bilayer, $L$ is the number of free lipids in solution (dictated from the CMC), $P$ is the number $n$ of monomeric proteins, $P_{n}$ is the number of protein $n$-mers, and $L P$ is the number of lipid-protein complexes (limited to a 1:1 stoichiometry in this simplified model). The amphiphilic aggregates undergo chemical equilibrium between amphiphilic molecules into aggregate and free molecules in the aqueous phase, where there is a continuous molecules exchange between solution and aggregate. On the one hand, the concentration of free lipids in solution is the CMC, and its value is a balance of hydrophobic acyl chains and polar head electrostatics. Long acyl chains contribute to a low CMC, whereas charged polar head groups favor a high CMC. On the other hand, the formation of unstructured soluble oligomers of the amyloidogenic proteins occurs if intermolecular interactions overcome intramolecular interactions ${ }^{55}$ and if the mechanism of oligomer formation shows a critical concentration due to "micelle-like" feature $^{56}$. Furthermore, alpha helix propensity of the PL complex might shift the equilibrium towards the complex. The formation of the various species in equilibria (a)-(c) depends on their related concentrations. Thus, by increasing either the free lipid $(L)$ or protein $(P)$ concentrations, the formation of chaperone-like $L P$ complexes must increase.

Our data suggest that the formation of amyloidogenic protein-lipid complex occurs in aqueous solution is dependent on free lipid concentration and transfers to the bilayer as a consequence of the chemical equilibrium. The proposed molecular mechanism is illustrated in Fig. 4. The popular amyloid hypothesis suggests that the damage to the membrane is due to the fibrillation on the membrane surface (a "detergent-like" mechanism). In contrast, the toxic oligomers hypothesis predicts that these soluble aggregates damage the membrane via ion-channel-like pores. The first hypothesis excludes the formation of pores, while the second hypothesis does not take the fibrillation process into account. The lipid-chaperone hypothesis is a more general molecular model that includes both hypotheses where the lipid-protein complex in solution plays the role of the main actor in membrane damage, and the molecular mechanism of membrane damage is a common factor of IDPs. In the following, we report three series of experiments that can be easily explained in the framework of the lipid chaperone hypothesis. 


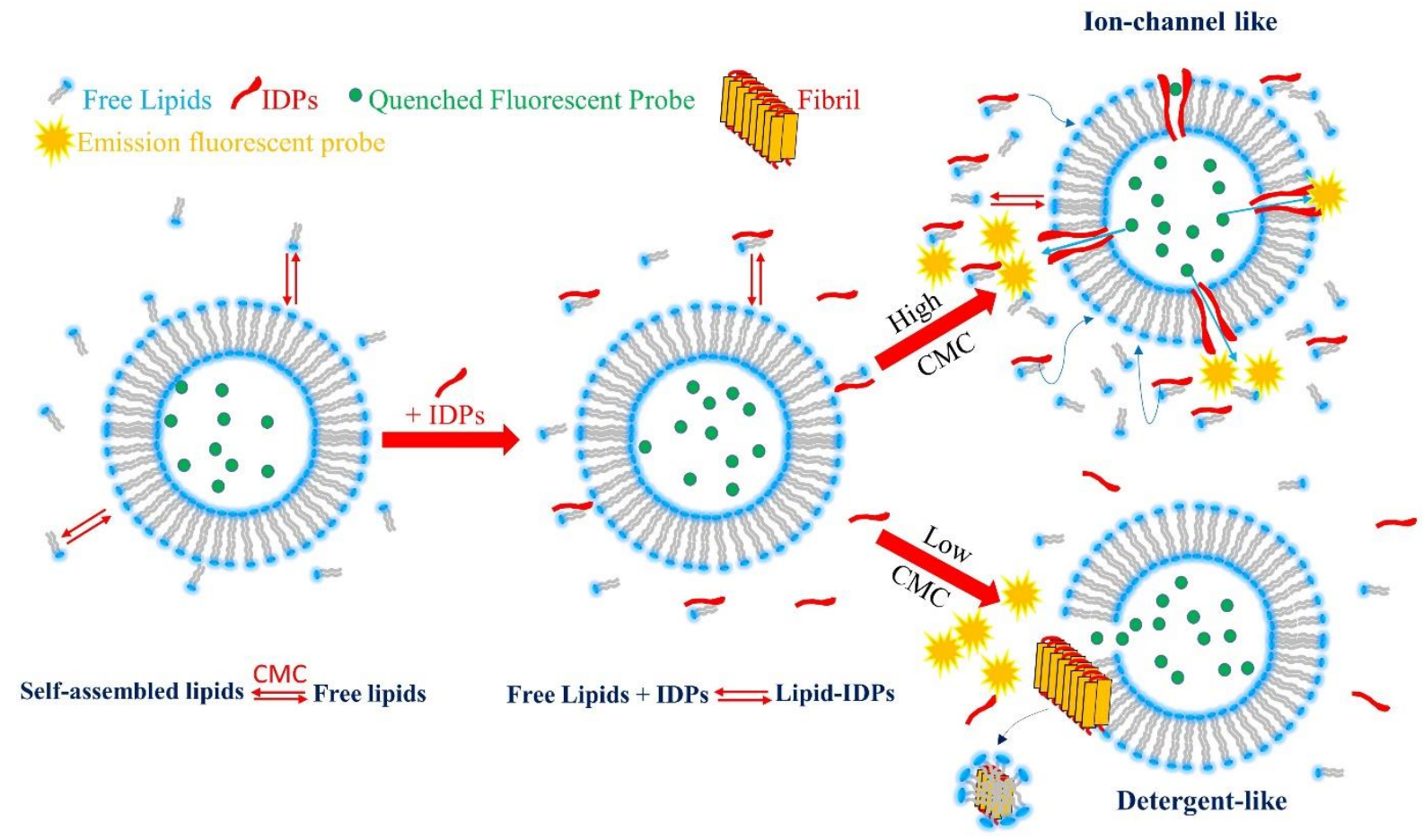

Figure 4. Schematic drawing illustrating how IDPs interact with a model membrane in the presence of free lipids at different CMC in the aqueous phase. Self-assembled lipids are in chemical equilibrium with free lipids in the aqueous phase (free lipids). There is a continuous exchange between self-assembled and free lipids. The concentration of free lipids remains constant over time (CMC). By the addition of IDPs, a stable lipid-IDPs complex is formed in the water phase. Two pathways can occur depending on the CMC values. A high CMC value favors ion-channel like pores, whereas a low CMC favors detergent-like mechanism. At intermediate CMC values, both mechanisms are feasible.

\section{Comparison to non-toxic and non-amyloidogenic proteins: rIAPP and $\beta$-syn}

To investigate whether this mechanism is limited to amyloidogenic and potentially toxic proteins, we examined the fluorescence-monitored leakage kinetics of two non-amyloidogenic proteins: $\beta$-syn and rat IAPP (rIAPP). Although rIAPP is not toxic at low concentration, it becomes toxic but remains non-amyloidogenic at $100 \mu \mathrm{M}^{18-22}$. (Ref to Brender et al Biochemistry) It has been shown that in the presence of LUVs composed of negatively charged phospholipids rIAPP does form pores ${ }^{23}$. In that paper, the authors concluded that there is no one-to-one relationship between the ability to induce leakage in model membranes and cytotoxicity, i.e. not all amyloidogenic IDPs that induce leakage are cytotoxic and not all amyloidogenic IDPs that are cytotoxic induce leakage. Here, we demonstrate that the relationship between cytotoxicity and the ability to induce leakage may be even more complex, because we can reverse the rIAPP behavior by varying the lipid composition. In Fig. 5A, we show the ThT fibril formation kinetics of rIAPP in the presence of LUVs composed by phospholipids with different acyl chain length (C14, C18 and C20). In agreement with literature 
reports, no fibril formation was observed for rIAPP, unlike hIAPP, $A \beta_{1-40}$, and $\alpha$-syn, which all form fibrils in the presence of LUVs formed composed of lipids with low CMC (see above). Fig. 5B shows the kinetics of carboxy-fluorescein leakage from LUVs in the presence of rIAPP.
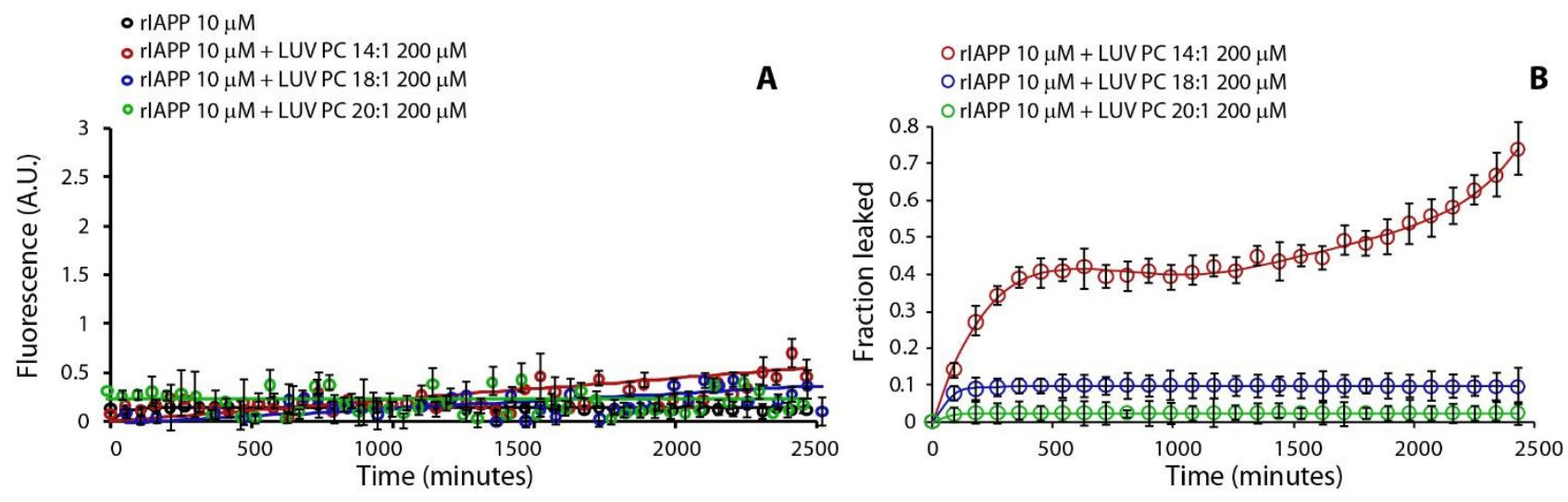

Figure 5. (A) Effect of lipid's hydrocarbon acyl chain length on rIAPP fibril formation kinetics (A) and dye-leakage (B). (A) ThT traces and (B) dye-leakage from samples containing rIAPP $10 \mu \mathrm{M}$ (black curve) in the presence of LUV PC 14:1 $200 \mu \mathrm{M}$ (red curve), LUV PC 18:1 $200 \mu \mathrm{M}$ (blue curve) and LUV PC 20:1 $200 \mu \mathrm{M}$ (green curve). Experiments were performed at $37{ }^{\circ} \mathrm{C}$ in 10 $\mathrm{mM}$ phosphate buffer, $100 \mathrm{mM} \mathrm{NaCl}, \mathrm{pH}$ 7.4. All results are the average of three experiments.

In order to further confirm these findings, we performed another test using $\beta$-syn interacting with LUVs containing different phospholipids (cite the figure for results). This 134 amino acid IDP is nontoxic and non-amyloidogenic ${ }^{24}$, which further confirms the difference between amyloidogenic and non-amyloidogenic proteins.

\section{The apparent role of bilayer thickness in membrane disruption by IDPs}

Bilayer thickness is strictly linked to CMC. Long chain phospholipids have low CMC and form thick bilayers, while short chain phospholipids have high CMC and form thinner bilayers. It has previously been shown that leakage resistance does not necessarily arise from a bilayer-protein mismatch, since the extent of pore formation by hIAPP in 22 PC LUVs can be dramatically increased by addition of 14 PC lipids at their CMC ${ }^{14,16,22}$. To examine whether this is the case for other IDPs, the same experiments performed with hIAPP was extended to $\alpha$-syn, $\beta$-syn, and rIAPP. Each of these proteins were incubated in a suspension of LUVs composed of phospholipids characterized by a very low CMC $22: 1 \mathrm{PC}\left(8 \times 10^{-10} \mathrm{M}\right)$ soluble $14: 1 \mathrm{PC}$ at a concentration of $1 \times 10^{-7}$ $\mathrm{M}$ (the CMC of this lipid). The very low concentration of $22: 1 \mathrm{PC}\left(8 \times 10^{-10} \mathrm{M}\right)$ in the aqueous phase is not sufficient to act as a chaperone for insertion of proteins into the bilayer, limiting the dye leakage. However, the addition of a relatively high concentration of free $14: 1 \mathrm{PC}\left(1 \times 10^{-7}\right)$ allows 
for the formation of the lipid-protein complex, promoting insertion into the bilayer, pore formation and increasing dye leakage. The results reported in Fig. 6 show that by the addition of short-chain high-CMC lipids $\alpha$-syn and rIAPP are capable of interacting with the 22:1 PC LUV membrane and forming pores, as was observed for hIAPP ${ }^{16}$. In contrast, non-toxic $\beta$-syn is not able to penetrate the membrane. This means that $\beta$-syn is not able to form a protein-lipid complex, probably because lack of hydrophobic NAC region in $\beta$-syn. These results clearly suggest the negligible role of membrane thickness while the high free lipid concentration acts as a carrier for proteins that penetrate the bilayer and form pores.

○ $\alpha$-synuclein $25 \mu \mathrm{M}+$ LUV PC22:1 $200 \mu \mathrm{M}+$ CMC PC 14:1

- $\beta$-synuclein $25 \mu \mathrm{M}+$ LUV PC22:1 $200 \mu \mathrm{M}+$ CMC PC 14:1

○ rlAPP $25 \mu \mathrm{M}+$ LUV PC22:1 $200 \mu \mathrm{M}+$ CMC PC 14:1

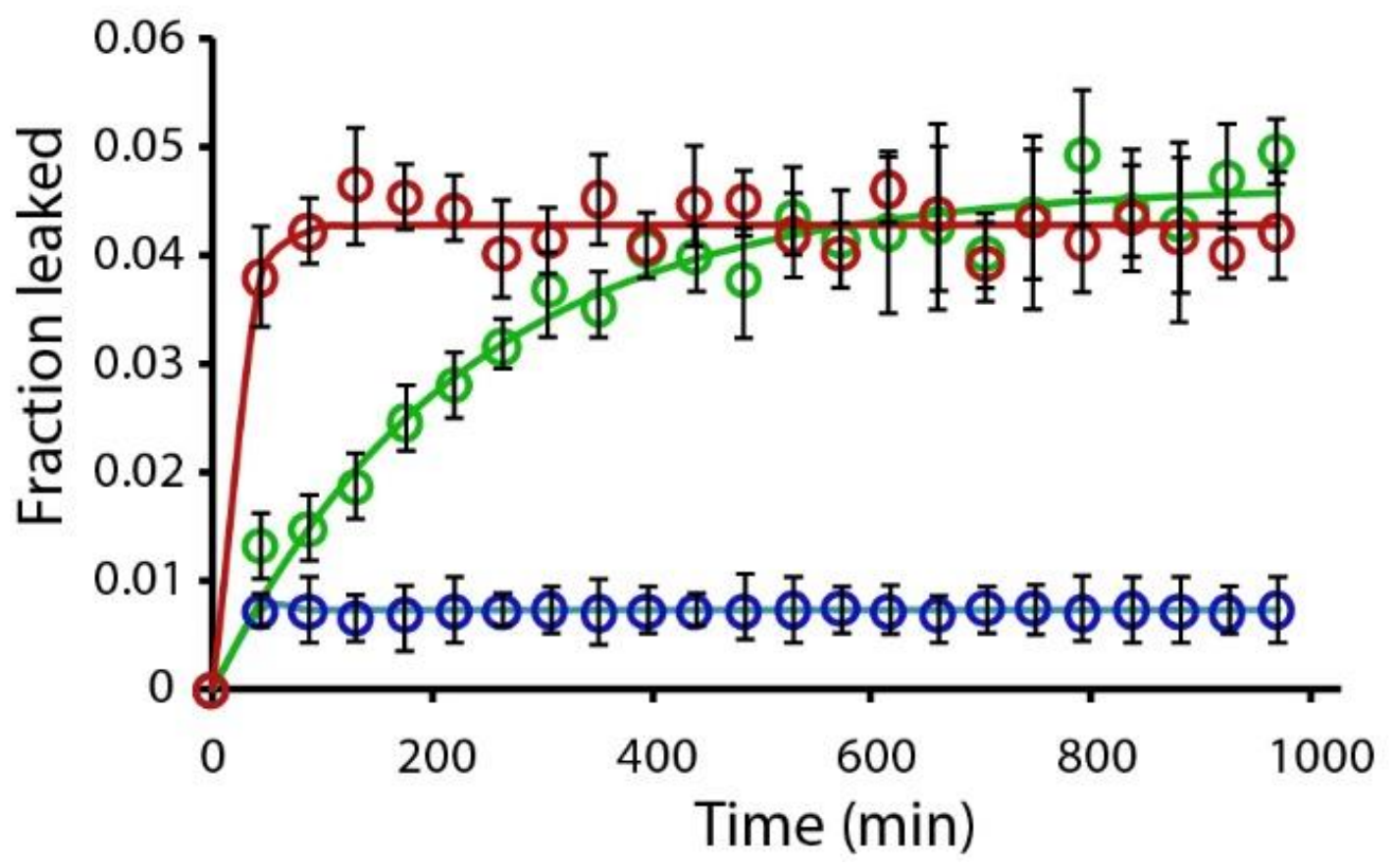

Figure 6. Effect of free PC 14:1 lipids on the disruption of LUVs of PC 22:1. Disruption of $200 \mu$ M LUVs of PC 22:1 induced by $25 \mu \mathrm{M} \alpha$-syn (red), $25 \mu \mathrm{M} \beta$-syn (blue) and $10 \mu \mathrm{M}$ rIAPP (green) in the presence of PC 14:1 at its CMC. Experiments were performed at $37^{\circ} \mathrm{C}$ in $10 \mathrm{mM}$ phosphate buffer, $100 \mathrm{mM} \mathrm{NaCl}, \mathrm{pH}$ 7.4. All results are the average of three experiments.

All the above-presented experimental data show the formation of protein-phospholipid complexes that play a major role in driving protein-membrane interactions. To further strengthen our hypothesis, we performed CD, 2D-NMR, ITC and all-atom molecular dynamics simulations to characterize the formation of the protein-lipid complex and to obtain structural information. 


\section{Is the role of Reactive Oxygen Species in disease progression related to generation of high CMC}

oxidized lipids?

We would like to conclude by testing our model on a totally different system. It is interesting to note that reactive oxygen species (ROS) have also been shown to play a role in type 2 diabetes, Alzheimer's and Parkinson's diseases-in particular by the oxidation of membrane phospholipids ${ }^{25-}$ ${ }^{34}$. ROS species derived from radicals operate at low concentrations in the cells. Their "steady state" concentration are determined by the balance between their rates of production and their rates of removal by various antioxidants (intracellular redox homeostasis or buffering). Under pathological conditions, redox homeostasis is unbalance so abnormally large concentrations of ROS are generated. Free ROS damage lipids, DNA and proteins. In particular, the oxidation of glycero-phospholipids produces a variety of oxidized lipids which show pathological and physiological relevance ${ }^{35}$. As they pertain to the lipid-chaperone model, oxidation of phospholipids significantly increases their CMC. For example, POPC has a CMC on the order of $\mathrm{nM}^{16}$ while the oxidation products of POPC have CMC values on the order of $\mu \mathrm{M}^{35}$. These dramatic changes in the CMC values may have important effects on the susceptibility of membranes to damage. If this hypothesis is correct, the susceptibility of model membranes composed of low-CMC phospholipids should increase in the presence of oxidative damaged lipids due to their higher CMC promoting membrane insertion of amyloidogenic proteins and leading to formation of ion-channel-like pores. Fig. 7 shows the effect of A $\beta_{1-40}$ on LUVs suspension containing PC22:1 and in the presence of oxidized PC22 at its CMC concentration. For PC22 it is expected to observe a very low poration following the trend shown in Fig. 2 (the CMC of PC22 is in the order of $\mathrm{nM}$ and the thickness of bilayer is greater than the other lipids examined in this work). However, the presence of oxidized lipids in the aqueous phase with a higher CMC leads to increased membrane poration induced by $\mathrm{A} \beta_{1-40}$, comparable with that observed for a shorter PC18 (Fig. 2). Conversely, for PC22 LUVs in the absence of oxidized lipids, the aqueous phase of high $\mathrm{CMC}$ remains stable, unaffected by the addition of $\mathrm{A} \beta_{1-40}$ as evidenced for hIAPP ${ }^{16}$. 


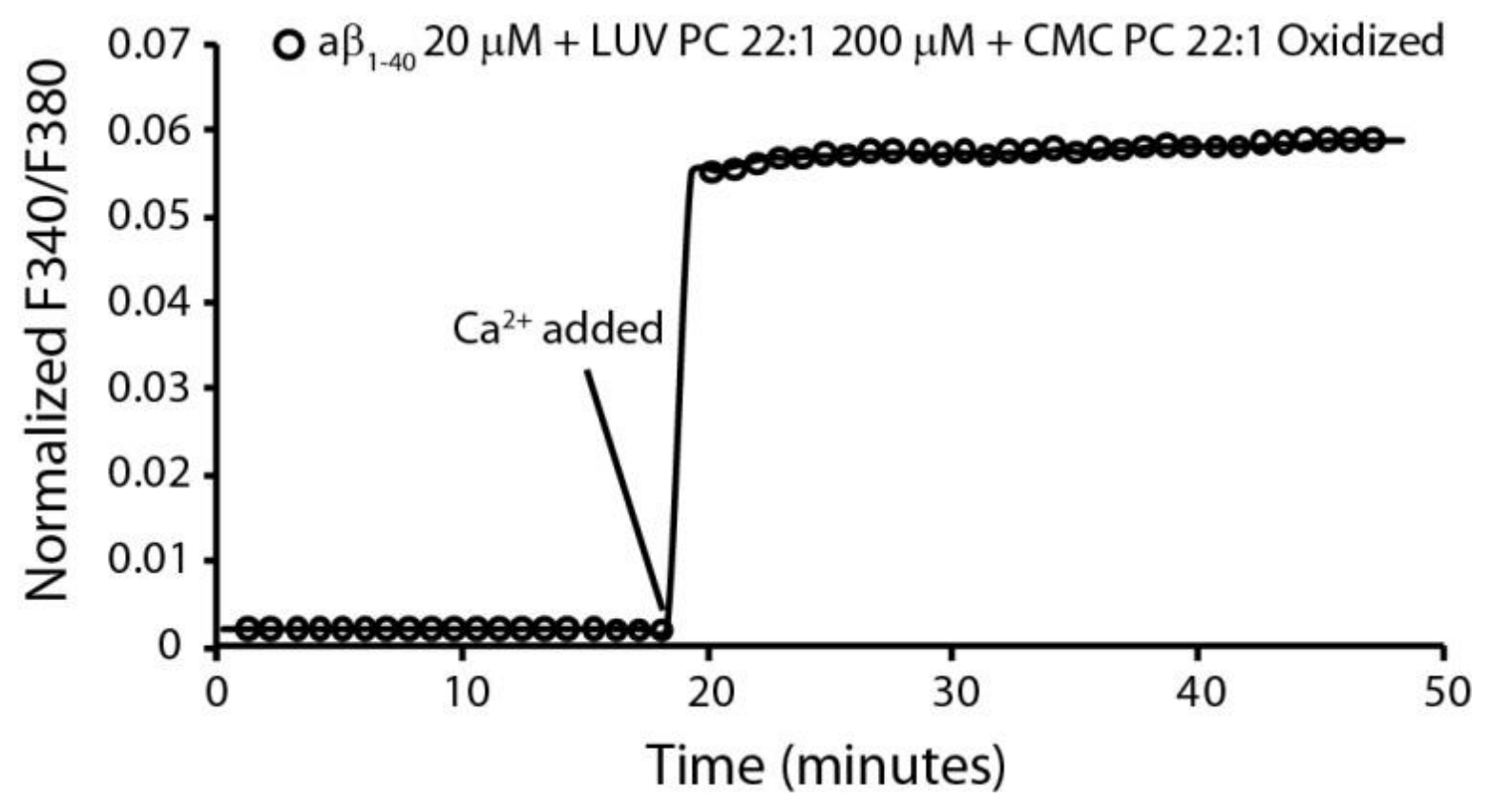

Figure 7. Oxidized lipids enhance the pore formation. For the experiment LUVs of PC 22:1 were prepared as described in materials and methods and hydrated by a solution of PC22:1 oxidized. Fura 2 experiment was performed as described in the material and methods section. Concentrations of A $\beta 1-40$, PC 22:1 LUVs, and oxidized PC 22:1 were 20, 200 and $1 \mu \mathrm{M}$ (its CMC), respectively.

The results obtained from this experiment suggest that oxidized lipids can act as chaperones forming the lipid-protein complex and thus making the protein suitable to penetrate the hydrocarbon core of the bilayer. The protein in the absence of free lipids having high CMC does not penetrate the membrane. The ability of lipids to act as a chaperone does not depend on its chemical structure, but rather on its concentration in the aqueous phase as highlights by our data.

Characterization of the protein-lipid complex by NMR and CD spectroscopies. 
The interaction between $\mathrm{A} \beta_{1-40}$ and PC 14:0 free lipids at CMC were monitored by 2D SOFASTHMQC NMR experiments at two different time intervals. The $2 \mathrm{D}{ }^{1} \mathrm{H} /{ }^{15} \mathrm{~N}$ correlation spectrum of $\mathrm{A} \beta_{1-40}$ incubated for $\sim 1$ hour at room temperature showed a substantial change in the chemical shifts and signal intensities (Fig. 8A). A reduction in signal intensities for most of the $A \beta_{1-40}$ residues was
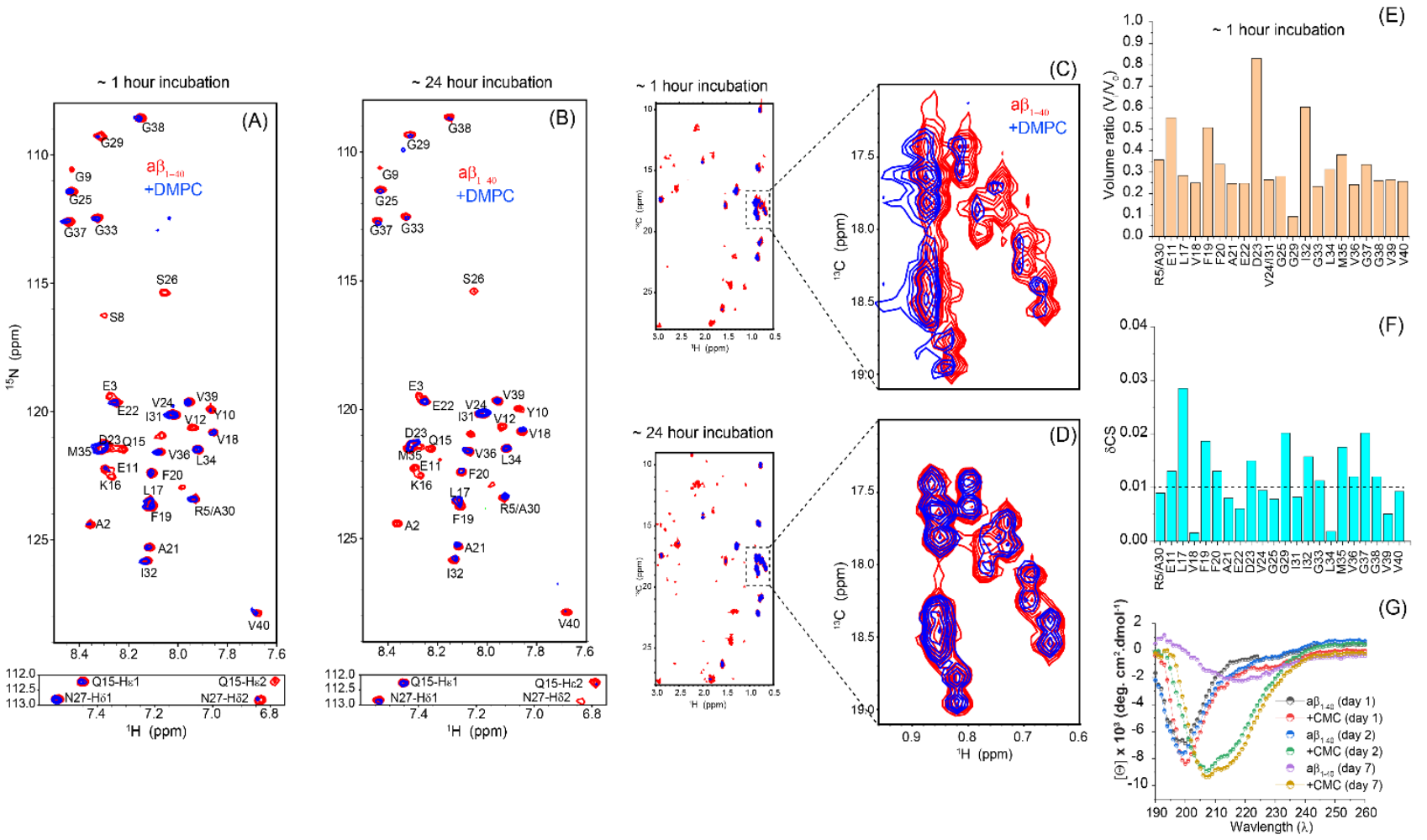

observed (Fig. 8E). Interestingly, at a time point of $~ 1$ hour, several peaks including A2, E3, S8, G9,

Figure 8. Interaction between $\mathbf{A} \beta_{1-40}$ and PC (14:0) free lipids at CMC. $2 \mathrm{D}{ }^{15} \mathrm{~N} /{ }^{1} \mathrm{H}$ SOFAST-HMQC spectra of $25 \mu \mathrm{M}$ freshly dissolved (in $10 \mathrm{mM}$ PBS, $\left.100 \mathrm{mM} \mathrm{NaCl}, 10 \% \mathrm{D}_{2} \mathrm{O}, \mathrm{pH}=7.4\right){ }^{15} \mathrm{~N} /{ }^{13} \mathrm{C}$ isotope-labelled $\mathrm{A} \beta_{1-40}$ in the absence (red) or presence (blue; plotted with 1.5x higher contour level as compared to the red spectrum) of lipids. NMR samples were measured at $\sim 1$ hour (A) and $\sim 24$ hours (B) of sample incubation at room temperature. All NMR spectra were recorded on an $800 \mathrm{MHz}$ Bruker spectrometer at 25 ${ }^{\circ} \mathrm{C}$ using a cryoprobe. The Gln/Asn sidechain N-H cross-peaks are shown below the corresponding 2D spectrum. Resonance assignment was adopted from previous studies ${ }^{36}(\mathrm{C}-\mathrm{D}) 2 \mathrm{D}{ }^{13} \mathrm{C} /{ }^{1} \mathrm{H}$ SOFAST-HMQC spectra of $\mathrm{A} \beta_{1-40}$ aliphatic region in the absence (red) or presence (blue) of PC (14:0) free lipids. The region spanning 17.0 to $19.0 \mathrm{ppm}$ in the ${ }^{13} \mathrm{C} /{ }^{1} \mathrm{H}$ spectrum is zoomed showing a reversible chemical shift perturbation over the time of incubation. (E) Graph shows the ratio of peak volume calculated from (A) where $V_{0}$ and $V_{i}$ represents the peak volumes of $\mathrm{A} \beta_{1-40}$ in the absence or presence of PC at CMC. (F) Combined chemical shift perturbations ( $\left.\delta \mathrm{CS}\right)$ in $A \beta_{1-40}$ calculated using the equation-1 (see methods) from spectrum (A). The dashed line presents the average chemical shift perturbations of all residues. $A \beta_{1-40}$ residues that show significant ${ }^{15} \mathrm{~N} /{ }^{1} \mathrm{H}$ line-broadening were excluded from the analysis in $(\mathrm{E}$ and F). (G) Time-lapse far-UV CD spectra of $25 \mu \mathrm{M}$ freshly dissolved (in $10 \mathrm{mM} \mathrm{PBS}, 100 \mathrm{mM} \mathrm{NaCl}, \mathrm{pH}=7.4$ ) unlabeled $\mathrm{A} \beta_{1-40}$ in the absence or presence of $\mathrm{PC}$ recorded at $25^{\circ} \mathrm{C}$ and at the indicated time points.

Y10, V12, Q15, K16, S26 and G29 were beyond the detection limit of NMR and are thus excluded from the analysis; it should be noted that the blue spectrum in Figure 6A was plotted with 1.5 times 
higher contour level as compared to the red spectrum in Sparky. The observed line-broadening indicates that the PC 14:0 free lipids at CMC interact selectively with the $\mathrm{N}$-terminal residues of $\mathrm{A} \beta_{1}$ 40; the depletion of S26 and G29 resonances is known to be substantially affected by temperature i.e. at $25^{\circ} \mathrm{C}^{37,38}$. At the same time, the uniform reduction in the peak intensities suggests the formation of a peptide-lipid complex that either tumbles slowly in the NMR time scale or possess conformational dynamics in the NMR time scale; but, the decrease in the signal intensities (or linebroadening) is not due to $A \beta_{1-40}$ fibrillation as ThT fluorescence showed no fluorescence at this timescale of aggregation (Fig. 1A). NMR spectra obtained from the sample incubated for $\sim 24$ hours at room temperature showed the presence of many well-resolved peaks unlike the spectrum obtained after $\sim 1$ hour incubation (Fig.8). These NMR results indicate that the conformation dynamics exhibited by the peptide-lipid complex at $\sim 1$ hour of incubation is likely stabilized at $\sim 24$ hours incubation, which results in the appearance of narrow lines at $\sim 24$ hours. In addition to the backbone amide signals, substantial line-broadening was observed in the sidechain $\mathrm{N} \delta-\mathrm{H} \delta 2$ and $\mathrm{N} \varepsilon-\mathrm{H} \varepsilon 2{ }^{15} \mathrm{~N} /{ }^{1} \mathrm{H}$ signals (Q15 and N27) indicating their involvement in lipid interactions. ${ }^{13} \mathrm{C} /{ }^{1} \mathrm{H}$ correlation spectra, as shown in Fig. 8C and 8D further identified the interaction of PC 14:0 free lipids at CMC to A $\beta_{1-40}$ side chain atoms. Notably, a significant chemical shift change was observed for the $A \beta_{1-40}$ aliphatic protons when incubated with free lipids for $\sim 1$ hour indicating a strong hydrophobic interaction. Surprisingly, after 24 hours of incubation, we did not observe any change in chemical shifts for the selected aliphatic region (Fig. 6D) indicating a spatial rearrangement of the lipids in the peptide-lipid complex. Combined chemical shift $(\delta C S)$ perturbation analysis derived from ${ }^{15} \mathrm{~N} /{ }^{1} \mathrm{H}$ SOFASTHMQC also showed a substantial $\delta C S$ with respect to the average CS for several residues as shown in Fig. 8E. This suggests PC 14:0 free lipids at CMC interaction induced a conformational change in $\mathrm{A} \beta_{1-40}$. This is further confirmed by time-lapse CD spectroscopy. $A \beta_{1-40}$ showed a disordered CD spectrum characterized by a CD minimum centered at $\approx 200 \mathrm{~nm}$ that follows a reduction in molar ellipticity [Ө] and red shift in the peak minimum on days 2 and 7 indicating peptide aggregation and $\beta$-sheet rich fibril formation (Fig. 8G). In contrast, PC 14:0 free lipids at CMC bound A $\beta_{1-40}$ presented an increase in $[\Theta]$ with two $\mathrm{CD}$ minima at $\approx 208$ and $\approx 215 \mathrm{~nm}$ on days 2 and 7 that closely resembles a $\alpha$-helix structure. Unlike $A \beta_{1-40}$ that forms fibril in the absence of lipids, $A \beta_{1-40}$ structure formation was found to be inhibited by the presence of free lipids for 7 days (Fig. 8G). This observation correlates with the ThT and NMR observations suggesting a delay in peptide aggregation coupled with complex formation, in $\mathrm{A} \beta_{1-40}$ incubated with PC 14:0 free lipids at CMC (Figs. 1A and 8F).

\section{Characterization of the protein-lipid complex: Molecular Dynamics simulations}


We employed all-atom (AA) molecular dynamics (MD) simulations to shed light, with atomistic details, on the structure-function relationship of four proteins (hIAPP ${ }^{39}$ [PDB ID: 2KB8], rIAPP ${ }^{40}$ [PDB ID: 2KJ7], A $\beta$ 1-40 and A $\beta$ 1-42 ${ }^{41}$ [PDB ID: 1Z0Q]) in complex with two lipid types: PC22 and PC14. The starting protein structures were taken from existing solution NMR structures deposited on the Protein Data Bank (see Materials and methods). We first stabilized the four proteins and the two lipids structures in a water solution for $500 \mathrm{~ns}$ under NpT conditions. The final frame of these simulations was employed to build the 1:1 protein-lipid systems, which have been simulated in triplicate for one microsecond each.

Since all proteins showed a stable binding with both lipid types after $100 \mathrm{~ns}$, a contact occupancy map has been calculated to study the average interaction patterns of protein-lipid complexes (Fig. 9A). Fig. 9A clearly shows a specific interaction between $A \beta_{1-40}, A \beta_{1-42}$, and hIAPP with both lipid types. The interaction pattern becomes more pronounced in the presence of PC22, which has longer lipid chains. These specific protein-lipid interactions take place through specific amino acid segments enriched with hydrophobic residues. By comparing the alpha helix propensity with the contact occupancy map, we noticed that the higher is the alpha-helix content, the more specific and strong is the binding. This agrees with the CD measurements showing conversion to the alpha-helix structure when a high concentration of free lipid is added to the amyloidogenic protein (Fig 8G). Indeed, structures showing high helix propensity have a specific binding between the acyl chain and the alpha helix region. When the helix propensity is low, the interaction pattern becomes broader and weaker (low probable). Our analysis led us to conclude that the protein-lipid complex is stabilized by hydrophobic interactions which are increased upon alpha-helix peptide folding. Interestingly, among the proteins studied, only rIAPP showed a very low helix propensity when interacting with lipids. That could be a critical difference between the behaviour of rIAPP and other amyloidogenic proteins. The hydrophobic nature of the protein-lipid complexes is explained by analysing the solventaccessible surface area (SASA) shown in Supplementary Figure S1-S4. Comparing the Supplementary Figure 1A-C, it is possible to see that the SASA of the complex is minor concerning the sum of protein and lipids individually. This result suggests the hydrophobic effect as the driving force for the formation of the complex for all the proteins. Overall, MD simulations showed a structure-dependent binding, pointing out the hydrophobic effect and helix propensity as a driving force for the lipid-protein complex.

We employed calorimetric measurements to shed light on the thermodynamic stability of the complex. ITC measurements were performed to determine the Gibbs free energy of formation for the phospholipid-protein complex. ITC data indicate a $\Delta \mathrm{G}_{\text {Bind }}$ value as high as $-63( \pm 20) \mathrm{kJ} \mathrm{mole}^{-1}$ (details in the ESI). The CD, MD, ITC, and NMR data are consistent with the model of a hydrophobic 
forces-driven lipid-protein complex as hypothesized in a previous theoretical model ${ }^{17}$. Also, the data suggest that lipid acts as a chaperone for the protein, driving the protein to penetrate the membrane as $\alpha$-helix as indicated by other authors ${ }^{40,42-45}$. 


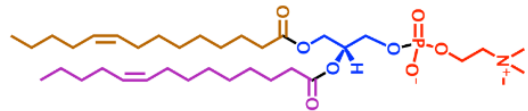

$A \beta_{1-40}$
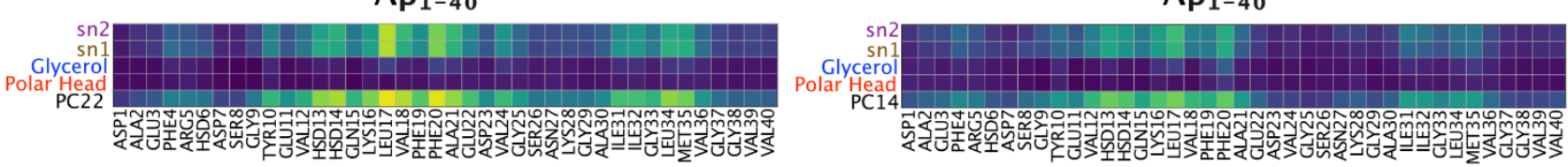

$A \beta_{1-42}$
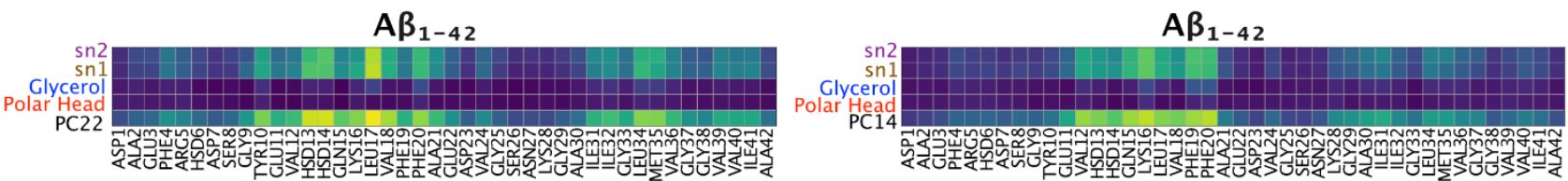

hIAPP
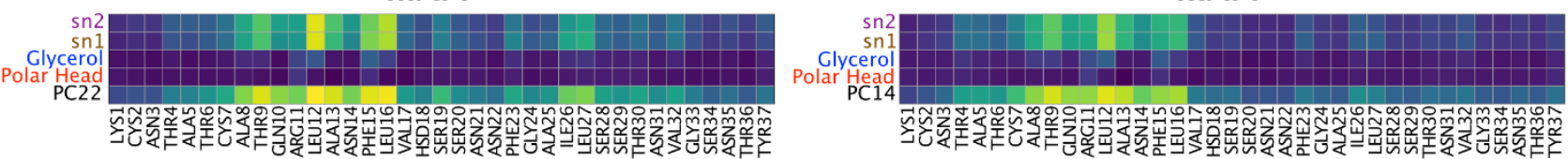

rIAPP
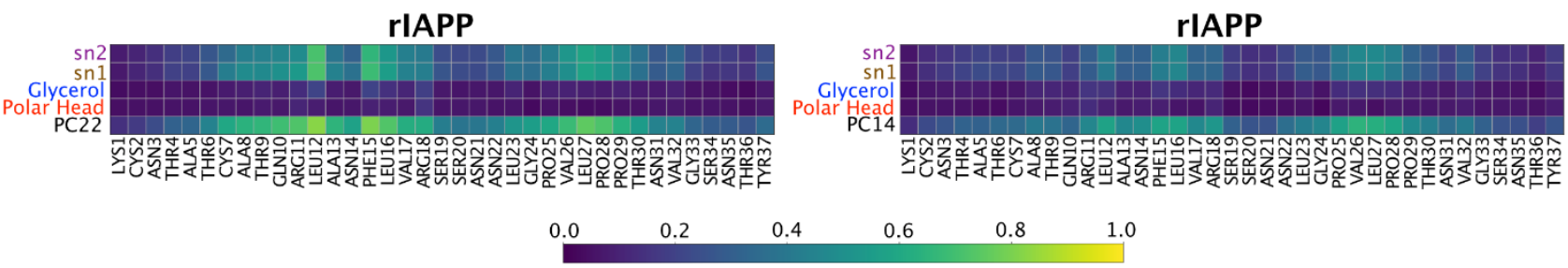

\section{Contact Occupancy}

B

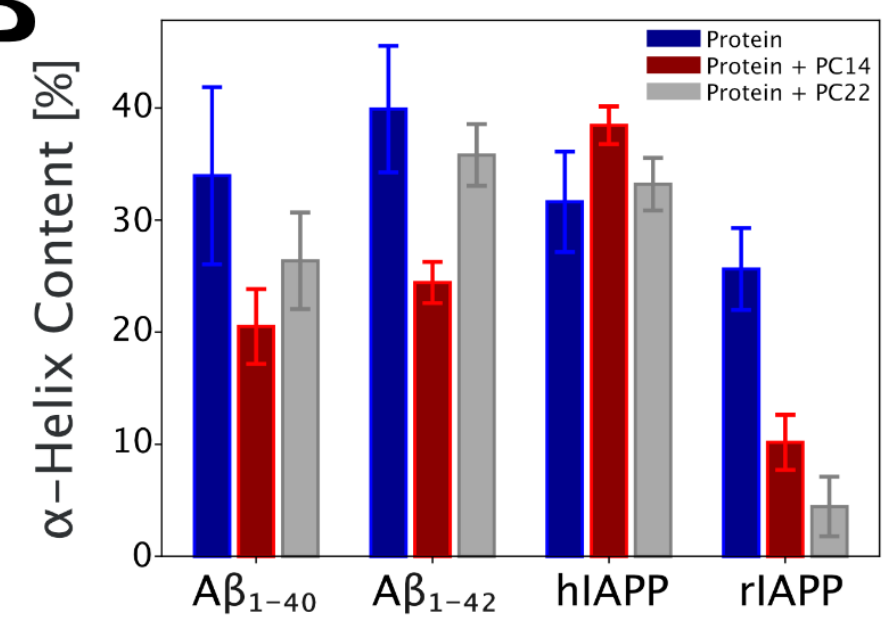

\section{hIAPP}
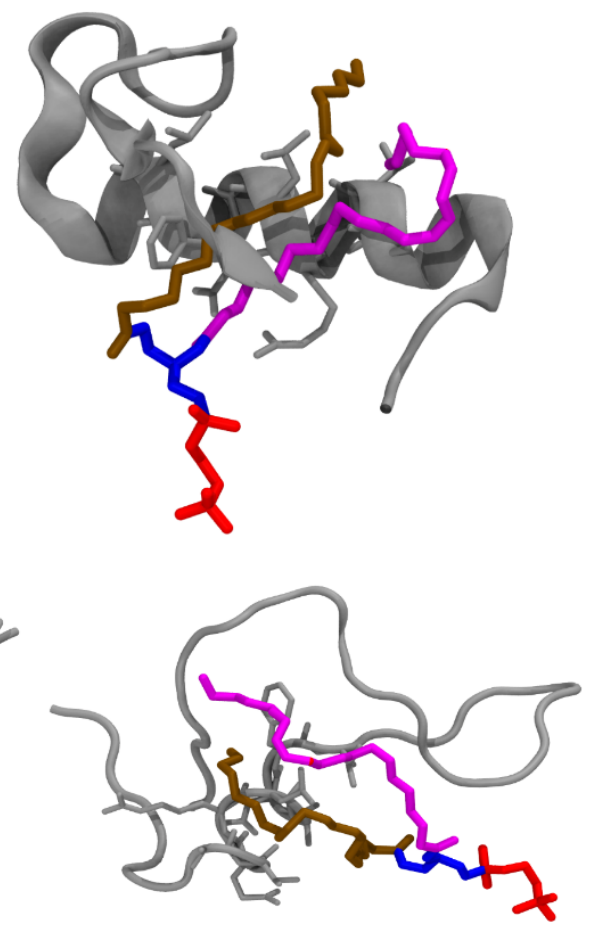

rIAPP 
Figure 9. Characterization of the molecular interface of the protein-lipid complexes based on atomistic molecular dynamics simulations. (A) Pairwise contact map with all residues for the protein-PC22 (left panel) and protein-PC14 (right) interfaces. Contact occupancy equal to 1.0 corresponds to the situation where a given interaction has taken place for the entire duration of the timeframe examined. The average was calculated by concatenating the last $900 \mathrm{~ns}$ from all the three repeats. A contact is defined if any of the atoms between two groups were closer than $0.6 \mathrm{~nm}$. (B) Structural analysis of protein's $\alpha$-helix content before and after the interaction with PC22 and PC14 lipids. The average is calculated over a trajectory $2.7 \mu$ s long obtained by concatenating the last $900 \mathrm{~ns}$ of each replica. The error bars represent the relative standard errors. Alongside shown are the corresponding 3D central structures of the protein-PC22 complexes. The central structure is the configuration with the smallest average RMSD from all other structures within the most populated cluster of the protein-PC22 interfaces. It is calculated by concatenating the last $100 \mathrm{~ns}$ of the three repeats.

The stability of the lipid-protein complex(es) is a fundamental prerequisite for the free lipids to act as a chaperone. This was proven by using different techniques as described above. The second mandatory condition is the greater hydrophobicity of the complex(es) relative to the bare protein. Indeed, only a large hydrophobicity guarantees a fast insertion of the assembly into the membrane core. By exploiting the lipid-protein contact maps of Fig. 9A and following the Eisenberg procedure $^{46}$, we calculated the hydrophobic index of bare proteins and lipid-protein complex(es) and included in the SI. These results unambiguously prove the enhanced hydrophobicity of the lipidprotein complexes as shown in Fig. 10.

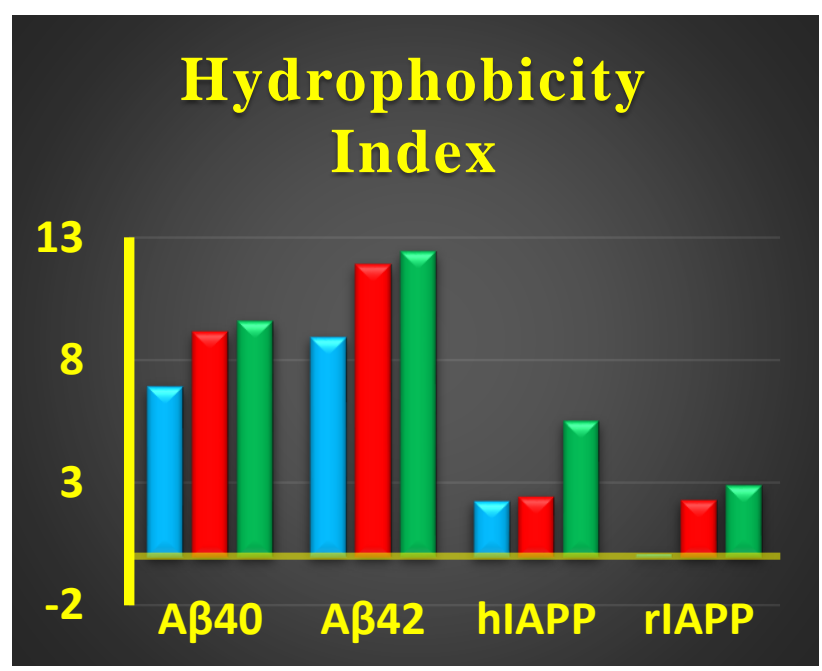

Figure 10. Hydrophobicity index of bare proteins (light blue), protein-DMPC (red, containing 14 carbon atoms per chain) and protein-DEPC (green containing 22 carbon atoms per chains).

\section{Discussion}

Recently, some of us have reported that the presence of free lipids with a high CMC (lipids containing short hydrocarbon chains) in solution suppresses the formation of fibrils by hIAPP and promotes the 
formation of pores in LUVs ${ }^{14}$. Lipids with very small CMC (long acyl chains) favor the detergentlike mechanism. Here, a similar behavior was confirmed for $A \beta_{1-40}$ and $\alpha$-syn, as reported in Figs. 1, 2 and 3, respectively. Data obtained from hIAPP, $A \beta_{1-40}$, and $\alpha$-syn share a common molecular mechanism in IDPs-membrane interaction and the concentration of free lipid in aqueous solution act as a switch between ion-channel like formation, detergent-like mechanism and fibril formation in the aqueous phase. We named the lipid-assisted transport of IDPs into bilayers the lipid-chaperone hypothesis. Moreover, detergent-like mechanisms and fibril formation in the bulk are not a correlated process. Fig. 1B shows the kinetics of damage to the membranes, using carboxy-fluorescein as fluorescent probe. In the case of $A \beta_{1-40}$, there is no pore formation detectable by carboxy-fluorescein, but only membrane damage by a detergent-like mechanism. Furthermore, as in the case of hIAPP, lipids with long hydrocarbon chains (low CMC) favour the detergent-like mechanism, while short acyl chain lipids (large $\mathrm{CMC}$ ) promote membrane damage via pore formation. In the case of $\mathrm{A} \beta$, the $\mathrm{Ca}^{2+}$-fura-2 complex must be used to detect the formation of ion-channel like pores, since their size does not allow the bulky carboxyfluorescein to cross the bilayer. Fig. 3 shows by adding calcium ions to LUVs containing fura 2 in their lumen previous incubated with $A \beta$, fluorescence increasing is due to $A \beta$-induced ion-channel like pores, thus calcium ions cross the membrane and form fluorescent fura $2 / \mathrm{Ca}^{++}$complex. Lipids with short acyl chains (low CMC) favours the intake of calcium in the lumen of LUVs, while the long hydrocarbon chains disfavour the poration mechanism. $\alpha$-Syn behaves like hIAPP and A $\beta$ since both the formation of fibrils and small pores are suppressed by large CMC lipids and favoured by the low CMC values (Fig. 2A and 2B). Furthermore, as in the case of hIAPP, $\mathrm{A} \beta_{1-40}$ and $\alpha$-syn both induce leakage in LUVs composed of intermediate chain length lipids by both pore-formation and detergent-like mechanisms.

In order to qualitatively examine whether the lipid-chaperone hypothesis could be relevant to in vivo cytotoxicity, the phospholipid acyl chain lengths, the phospholipase activity, and the oxidative stress in people affected by type 2 diabetes, Alzheimer's and Parkinson's diseases, can be compared with those of healthy population. It has been reported that patients with diabetes mellitus type 2 show higher concentrations of polar phospholipids in plasma with respect to those of healthy people. In particular, the concentration of sphingolipids with hydrocarbon chains longer than C18 increases ${ }^{26}$. Moreover, phospholipids with unsaturated or short fatty acids (high CMC) were concentrated in granules where IAPP is co-secreted with insulin ${ }^{27}$. Lastly, it was shown that diabetes is characterized by an increased concentration of saturated acyl-chain phospholipids and a decrease of unsaturated chains, which have higher CMC values ${ }^{28}$. This trend was also noticed in an age-matched comparison affected by Alzheimer's and Parkinson's diseases ${ }^{29-33}$. Based on these observations, a lipidomic approach to detect preclinical Alzheimer's disease was proposed in a group of cognitively normal 
older adults ${ }^{34}$. In addition, Type 2 diabetes mellitus, Alzheimer's and Parkinson's diseases share other common clinical features including higher phospholipase activity, which concurrently leads to the generation of higher CMC phospholipids ${ }^{35-43}$.

\section{Conclusion}

The lipid-chaperone hypothesis stems by considering that amphiphilic aggregates undergo chemical equilibrium between amphiphilic molecules into aggregate and free molecules in the aqueous phase, where there is a continuous amphiphilic molecules exchange between solution and aggregate. The concentration of free lipids in solution is the CMC, and its value is a balance of hydrophobic acyl chains and polar head electrostatics. Long acyl chains contribute to a low CMC, whereas charged polar head groups favour a high CMC. On the other hand, the formation of unstructured soluble oligomers of amyloidogenic proteins occurs if intermolecular interactions overcome intramolecular interactions ${ }^{47}$ and the mechanism of oligomer formation shows a critical concentration due to "micelle-like" feature ${ }^{48}$. Our data suggest that the formation of amyloidogenic protein-lipid complex occurs in aqueous solution is dependent on free lipid concentration and transfers to the bilayer as a consequence of the chemical equilibrium. The proposed molecular mechanism is illustrated in Fig. 4. The amyloid hypothesis suggests that the damage to the membrane is due to the fibrillation on the membrane surface (a "detergent-like" mechanism), whereas the toxic oligomers hypothesis predicts that these soluble aggregates damage the membrane via ion-channel-like pores. The first hypothesis excludes the formation of pores, while the second hypothesis does not take the fibrillation process into account. The lipid-chaperone hypothesis is a more general molecular model that includes both hypotheses where the lipid-protein complex plays the role of the main actor in membrane damage, and the molecular mechanism of membrane damage is a common factor of IDPs. The lipid-chaperone hypothesis suggests new routes to explore the development of effective drugs and suggests that oxidation of membrane phospholipids may be a likely route.

\section{Methods}

Materials and methods are available on Electronic Supplementary information. 


\section{References}

1 D. J. Selkoe and J. Hardy, EMBO Mol. Med., 2016, 8, 595-608.

2 J. A. Hebda and A. D. Miranker, Annu. Rev. Biophys., 2009, 38, 125-152.

3 F. Chiti and C. M. Dobson, Annu. Rev. Biochem., 2006, 75, 333-366.

4 N. Arispe, E. Rojas and H. B. Pollard, Proc. Natl. Acad. Sci., 1993, 90, 567-571.

5 S. M. Butterfield and H. A. Lashuel, Angew. Chem. Int. Ed., 2010, 49, 5628-5654.

6 F. Chiti and C. M. Dobson, Annu. Rev. Biochem., 2017, 86, 27-68.

7 M. F. M. Sciacca, S. A. Kotler, J. R. Brender, J. Chen, D. Lee and A. Ramamoorthy, Biophys. J., 2012, 103, 702-710.

8 S. Scalisi, M. F. M. Sciacca, G. Zhavnerko, D. M. Grasso, G. Marletta and C. La Rosa, ChemBioChem, 2010, 11, 1856-1859.

9 A. Quist, I. Doudevski, H. Lin, R. Azimova, D. Ng, B. Frangione, B. Kagan, J. Ghiso and R. Lal, Proc. Natl. Acad. Sci. U. S. A., 2005, 102, 10427-10432.

10 H. Jang, F. T. Arce, S. Ramachandran, R. Capone, R. Azimova, B. L. Kagan, R. Nussinov and R. Lal, Proc. Natl. Acad. Sci., 2010, 107, 6538-6543.

11 M. Pannuzzo, A. Raudino, D. Milardi, C. La Rosa and M. Karttunen, Sci. Rep., 2013, 3, 2781.

12 J. Zhao, R. Hu, M. F. M. Sciacca, J. R. Brender, H. Chen, A. Ramamoorthy and J. Zheng, Phys. Chem. Chem. Phys., 2014, 16, 2368-2377.

13 B. D. van Rooijen, M. M. A. E. Claessens and V. Subramaniam, Biochim. Biophys. Acta BBA - Biomembr., 2009, 1788, 1271-1278.

14 K. J. Korshavn, C. Satriano, Y. Lin, R. Zhang, M. Dulchavsky, A. Bhunia, M. I. Ivanova, Y.-H. Lee, C. La Rosa, M. H. Lim and A. Ramamoorthy, J. Biol. Chem., 2017, 292, 4638-4650.

15 D. Marsh, Biophys. J., 2012, 102, 1079-1087.

16 F. Scollo, C. Tempra, F. Lolicato, M. F. M. Sciacca, A. Raudino, D. Milardi and C. La Rosa, J. Phys. Chem. Lett., 2018, 9, 5125-5129.

17 C. La Rosa, S. Scalisi, F. Lolicato, M. Pannuzzo and A. Raudino, J. Chem. Phys., 2016, 144, 184901.

18 K. Korshavn, C. Satriano, R. Zhang, Y. Lin, M. Dulchavsky, A. Bhunia, M. Ivanova, Y. Lee, C. La Rosa, M. Lim and others, Mech. Struct. Insights Chem. Modul. Amyloid Aggreg., 2017, 105.

19 M. F. Tomasello, A. Sinopoli, F. Attanasio, M. L. Giuffrida, T. Campagna, D. Milardi and G. Pappalardo, Eur. J. Med. Chem., 2014, 81, 442-455.

20 M. Magzoub and A. D. Miranker, FASEB J., 2012, 26, 1228-1238.

21 R. P. R. Nanga, J. R. Brender, J. Xu, G. Veglia and A. Ramamoorthy, Biochemistry, 2008, 47, 12689-12697.

22 K. J. Korshavn, A. Bhunia, M. H. Lim and A. Ramamoorthy, Chem. Commun., 2016, 52, 882-885.

23 P. Cao, A. Abedini, H. Wang, L.-H. Tu, X. Zhang, A. M. Schmidt and D. P. Raleigh, Proc. Natl. Acad. Sci., 2013, 110, 19279-19284.

24 C. Di Scala, N. Yahi, S. Boutemeur, A. Flores, L. Rodriguez, H. Chahinian and J. Fantini, Sci. Rep., 2016, 6, 28781.

25 W. Ahmad, B. Ijaz, K. Shabbiri, F. Ahmed and S. Rehman, J. Biomed. Sci., 2017, 24, 76.

26 A. P. Gunn, B. X. Wong, T. Johanssen, J. C. Griffith, C. L. Masters, A. I. Bush, K. J. Barnham, J. A. Duce and R. A. Cherny, J. Biol. Chem., 2016, 291, 6134-6145.

27 A. W. Pilkington, G. C. Donohoe, N. G. Akhmedov, T. Ferrebee, S. J. Valentine and J. Legleiter, Biochemistry, 2019, 58, 2893-2905.

28 S. C. Dyall, Int. J. Alzheimers Dis., 2010, 2010, 1-10.

29 U. Asmat, K. Abad and K. Ismail, Saudi Pharm. J., 2016, 24, 547-553.

30 M. P. Mattson, W. A. Pedersen, W. Duan, C. Culmsee and S. Camandola, Ann. N. Y. Acad. Sci., 1999, 893, 154-175.

31 L. Lyras, N. J. Cairns, A. Jenner, P. Jenner and B. Halliwell, J. Neurochem., 2002, 68, 2061-2069.

32 L. Puspita, S. Y. Chung and J. Shim, Mol. Brain, 2017, 10, 53.

33 Z. Wei, X. Li, X. Li, Q. Liu and Y. Cheng, Front. Mol. Neurosci., 2018, 11, 236.

34 E. Wright, J. L. Scism-Bacon and L. C. Glass, Int. J. Clin. Pract., 2006, 60, 308-314.

35 A. H. Pande, S. Kar and R. K. Tripathy, Biochim. Biophys. Acta BBA - Biomembr., 2010, 1798, 442-452. 
36 B. R. Sahoo, T. Genjo, T. W. Nakayama, A. K. Stoddard, T. Ando, K. Yasuhara, C. A. Fierke and A.

Ramamoorthy, Chem. Sci., 2019, 10, 3976-3986.

37 T. Yamaguchi, K. Matsuzaki and M. Hoshino, FEBS Lett., 2011, 585, 1097-1102.

38 B. R. Sahoo, T. Genjo, S. J. Cox, A. K. Stoddard, G. M. Anantharamaiah, C. Fierke and A. Ramamoorthy, J. Mol. Biol., 2018, 430, 4230-4244.

39 S. M. Patil, S. Xu, S. R. Sheftic and A. T. Alexandrescu, J. Biol. Chem., 2009, 284, 11982-11991.

40 R. P. R. Nanga, J. R. Brender, J. Xu, K. Hartman, V. Subramanian and A. Ramamoorthy, J. Am. Chem. Soc., 2009, 131, 8252-8261.

41 S. Tomaselli, V. Esposito, P. Vangone, N. A. J. van Nuland, A. M. J. J. Bonvin, R. Guerrini, T. Tancredi, P. A. Temussi and D. Picone, ChemBioChem, 2006, 7, 257-267.

42 M. F. M. Engel, H. Yigittop, R. C. Elgersma, D. T. S. Rijkers, R. M. J. Liskamp, B. de Kruijff, J. W. M. Höppener and J. Antoinette Killian, J. Mol. Biol., 2006, 356, 783-789.

43 L. Khemtémourian, M. F. M. Engel, J. A. W. Kruijtzer, J. W. M. Höppener, R. M. J. Liskamp and J. A. Killian, Eur. Biophys. J., 2010, 39, 1359-1364.

44 N. C. Kegulian, S. Sankhagowit, M. Apostolidou, S. A. Jayasinghe, N. Malmstadt, P. C. Butler and R. Langen, J. Biol. Chem., 2015, jbc.M115.659797.

45 S. Vivekanandan, J. R. Brender, S. Y. Lee and A. Ramamoorthy, Biochem. Biophys. Res. Commun., 2011, 411, 312-316.

46 D. Eisenberg, E. Schwarz, M. Komaromy and R. Wall, J. Mol. Biol., 1984, 179, 125-142.

47 C. La Rosa, M. Condorelli, G. Compagnini, F. Lolicato, D. Milardi, T. N. Do, M. Karttunen, M. Pannuzzo, A. Ramamoorthy, F. Fraternali, F. Collu, H. Rezaei, B. Strodel and A. Raudino, Eur. Biophys. J., 2020, 49, 175-191.

48 J. R. Brender, J. Krishnamoorthy, M. F. M. Sciacca, S. Vivekanandan, L. D’Urso, J. Chen, C. La Rosa and A. Ramamoorthy, J. Phys. Chem. B, 2015, 119, 2886-2896.

\section{Acknowledgements}

Research in the Ramamoorthy lab is supported by funds from NIH (AG048934 to A.R.). JCL and MDW are supported by the Intramural Research Program at the National Institutes of Health, National Heart, Lung, and Blood Institute. CLR are supported by University of Catania, grant PiCeRi 202022.

\section{Corresponding authors}

Carmelo La Rosa, clarosa@unict.it 